\title{
THE SIMILARITY PROBLEM FOR INDEFINITE STURM-LIOUVILLE OPERATORS WITH PERIODIC COEFFICIENTS
}

\author{
Aleksey Kostenko
}

Abstract. We investigate the problem of similarity to a self-adjoint operator for $J$-positive SturmLiouville operators $L=\frac{1}{\omega}\left(-\frac{d^{2}}{d x^{2}}+q\right)$ with $2 \pi$-periodic coefficients $q$ and $\omega$. It is shown that if 0 is a critical point of the operator $L$, then it is a singular critical point. This gives us a new class of $J$-positive differential operators with the singular critical point 0 . Also, we extend the Beals and Parfenov regularity conditions for the critical point $\infty$ to the case of operators with periodic coefficients.

Mathematics subject classification (2010): 47E05, 34B24, 34B09, 34L10, 47B50.

Keywords and phrases: $J$-self-adjoint operator, Sturm-Liouville operator, similarity, critical points.

\section{REFERENCES}

[1] R. BEALS, Indefinite Sturm-Liouville problems and half-range completeness, J. Differential Equations 56 (1985), 391-407.

[2] J. Behrndt And C. TRUnK, On the negative squares of indefinite Sturm-Liouville operators, J. Differential Equations 238 (2007), 491-519.

[3] P. Binding And B. ĆURgus, A counterexample in Sturm-Liouville completeness theory, Proc. Roy. Soc. Edinburgh Sect. A 134, 2 (2004), 244-248.

[4] M.S. BIRMAN AND M.Z. SOlOMJAK, Spectral theory of self-adjoint operators in Hilbert spaces, D. Reidel Publ. Company, Dordrecht, 1987.

[5] B. ĆURGUS, On the regularity of the critical point infinity of definitizable operators, Integr. Equ. Oper. Theory 8 (1985), 462-488.

[6] B. ĆURGUS, Positive operators in Krein spaces similar to self-adjoint operators in Hilbert spaces, (2006), http://www.math.tu-berlin.de/ trunk/abstracts6.html.

[7] B. ĆURGUS AND H. LANGER, A Krein space approach to symmetric ordinary differential operators with an indefinite weight function, J. Differential Equations 79 (1989), 31-61.

[8] B. ĆURGUS AND B. NAJMAN, The operator $(\operatorname{sgn} x) \frac{d^{2}}{d x^{2}}$ is similar to a selfadjoint operator in $L^{2}(\mathbb{R})$, Proc. Amer. Math. Soc. 123 (1995), 1125-1128.

[9] K. DAho AND H. LANGER, Sturm-Liouville operators with an indefinite weight function: the periodic case, Radovi Mat. 2 (1986), 165-188.

[10] A. FleIGE, Spectral theory of indefinite Krein-Feller differential operators, Mathematical Research 98, Berlin, Akademie Verlag, 1996.

[11] A. FLEIGE, A necessary aspect of the generalized Beals condition for the Riesz basis property of indefinite Sturm-Liouville problems, Oper. Theory: Adv. Appl. 175 (2007), 89-94.

[12] A. FleIge, The Riesz basis property of indefinite Sturm-Liouville problems with non-odd weight function, Integr. Equ. Oper. Theory 60 (2008), 237-246.

[13] I.M. GELFAND, Expansion in characteristic functions of an equation with periodic coefficients, Doklady Akad. Nauk SSSR 73 (1950), 1117-1120. (Russian)

[14] F. GESZTESY AND V. TKACHENKO, A criterion for Hill operators to be spectral operators of scalar type, J. Anal. Math. 107 (2009), 287-353.

[15] I.M. KARABASH, Abstract kinetic equations with positive collision operators, Oper. Theory: Adv. Appl. 188 (2008), 175-195. 
[16] I.M. KARABASH, A functional model, eigenvalues, and finite singular critical points for indefinite Sturm-Liouville operators, Oper. Theory: Adv. Appl. 203 (2009), 247-287.

[17] I.M. KARABASH AND A.S. KOSTENKO, Indefinite Sturm-Liouville operators with the singular critical point zero, Proc. Roy. Soc. Edinburgh Sect. A 138 (2008), 801-820.

[18] I.M. KARABASH AND A.S. KostenKo, On the similarity of a J-nonnegative Sturm-Liouville operator to a selfadjoint operator, Funct. Anal. Appl. 43 (2009), 65-68.

[19] I.M. Karabash, A.S. Kostenko, And M.M. Malamud, The similarity problem for $J$ nonnegative Sturm-Liouville operators, J. Differential Equations 246 (2009), 964-997.

[20] I.M. Karabash AND M.M. Malamud, Indefinite Sturm-Liouville operators $(\operatorname{sgn} x)\left(-\frac{d^{2}}{d x^{2}}+q\right)$ with finite-zone potentials, Oper. Matrices 1, 3 (2007), 301-368.

[21] T. Kato, Perturbation theory for linear operators, Springer-Verlag, Berlin-Heidelberg, New York, 1966.

[22] M. G. KREIN, The theory of self-adjoint extensions of semi-bounded Hermitian transformations and its applications, II, Mat. Sb. (N.S.) 21, 62 (1947), 365-404. (Russian)

[23] H. LANGER, Spectral functions of definitizable operators in Krein space, Lecture Notes in Math. 948 (1982), 1-46.

[24] M.M. MALAMUd, A criterion for the similarity of a closed operator to a self-adjoint operator, Ukrainian Math. J. 37 (1985), 41-48.

[25] D. MCGarvey, Operators commuting with translation by one. I. Representation theorems, J. Math. Anal. Appl. 4 (1965), 366-410.

[26] S.N. NAвоко, Conditions for similarity to unitary and self-adjoint operators, Funct. Anal. Appl. 18, 1 (1984), 13-22.

[27] M.A. NAIMARK, Linear differential operators, Fizmatgiz, Moscow, 1969. (Russian)

[28] A.I. PARFENOv, On an embedding criterion for interpolation spaces and application to indefinite spectral problem, Siberian Math. J. 44, 4 (2003), 638-644.

[29] S.G. Pyat Kov, Some properties of eigenfunctions of linear pencils, Math. Notes 51 (1992), 90-95.

[30] S.G. Pyatkov, Some properties of eigenfunctions and associated functions of indefinite SturmLiouville problems, in: Nonclassical Problems of Mathematical Physics, Sobolev Institute of Mathematics, Novosibirsk (2005), 240-251.

[31] M. REed AND B. Simon, Methods of modern mathematical physics, IV: Analysis of operators, Academic Press, New York, 1978.

[32] F.S. Rofe-BeKetov AND A.M. KholKin, Spectral analysis of differential operators: interplay between spectral and oscillatory properties, World Scientific Publishing Co. Pte. Ltd., Hackensack, NJ, 2005.

[33] E.C. Titchmarsh, Eigenfunction expansions associated with second-order differential equations. II, Clarendon Press, Oxford, 1958.

[34] O.A. Veliev, Spectrum and spectral singularities of differential operators with complex valued periodic coefficients, Diff. Equats. 19 (1983), 983-989.

[35] K. VESELIĆ, On spectral properties of a class of J-self-adjoint operators. I, Glasnik Math. Ser. III 7, 2 (1972), 229-248.

[36] H. VolKmer, Sturm-Liouville problems with indefinite weights and Everitt's inequality, Proc. Roy. Soc. Edinburgh Sect. A 126 (1996), 1097-1112.

[37] J. Weidmann, Spectral theory of ordinary differential operators, Lecture Notes in Math. 1258, 1987.

[38] A. ZettL, Sturm-Liouville theory, Amer. Math. Soc., Providence, RI, 2005. 